\title{
Widespread Necrotizing Purpura and Lucio Phenomenon as the First Diagnostic Presentation of Diffuse Nonnodular Lepromatous Leprosy
}

\author{
Amira Shahin, MD; Randa Yousef, MD; Soliman Hussein, MD; Marwa Niazy, MD; \\ Doaa H.S. Attia, MD; Maha F. Elmasry, MD; Suzan Shalaby, MD; Heba A. Abdelkader, MD; \\ Menna Bahgat, MBBCh, MSc; Doaa Teleb, MBBCh, MSc; Amira Elbendary, MBBCh, MSc; \\ Ahmed Mourad, MBBCh, MSc; Samar Hamed, MBBCh; Mona R.E. Abdel-Halim, MD
}

\section{PRACTICE POINTS}

- Leprosy is a great mimicker of many connective tissue diseases, including vasculitis.

- Antiphospholipid antibodies are involved in Lucio phenomenon.

- Prompt treatment is important in Lucio phenomenon to avoid morbidity and mortality.

Lucio phenomenon, or Lucio leprosy, is a rare severe lepra reaction that develops exclusively in patients with diffuse nonnodular lepromatous leprosy. It is characterized by irregular, angulated, or stellar necrotizing purpuric lesions that develop ulcerations. It mainly involves the extremities and develops as a result of massive invasion of vascular endothelial cells with lepra bacilli and secondary thrombotic vascular occlusion. Antiphospholipid antibodies often are detected in cases of Lucio phenomenon, and they are thought to play a role in its pathogenesis. We report a case of diffuse lepromatous leprosy in Egypt in which Lucio phenomenon with scrotal involvement and positive antiphospholipid antibodies was the first diagnostic presentation. The patient showed an excellent response to a combination of antileprotic treatment, low dose of prednisolone, acetylsalicylic acid, and anticoagulants. In addition, surgical debridement and vacuum therapy were performed for the scrotal lesion. Awareness of this grave presentation of leprosy is important for both dermatologists and rheumatologists to avoid misdiagnosis as vasculitis/collagen disease.

Cutis. 2021;108:E4-E8.

\section{Case Report}

A 70-year-old man living in Esna, Luxor, Egypt presented to the Department of Rheumatology and Rehabilitation with widespread gangrenous skin lesions associated with ulcers of 2 weeks' duration. One year prior, the patient had an insidious onset of nocturnal fever, bilateral leg edema, and numbness and a tingling sensation in both hands. He presented some laboratory and radiologic investigations that were performed at another hospital prior to the current presentation, which revealed thrombocytopenia, mild splenomegaly, and generalized lymphadenopathy. An excisional left axillary lymph node biopsy was performed at another hospital prior to the current presentation, and the pathology report provided by the patient described a reactive, foamy, histiocyterich lesion, suggesting a diagnosis of hemophagocytic

Drs. Shahin, Yousef, Niazy, Attia, Elmasry, Shalaby, Abdelkader, and Abdel-Halim as well as Ms. Teleb, Ms. Elbendary, Mr. Mourad, and Mr. Hamed are from the Kasr Al Aini Hospital, Faculty of Medicine, Cairo University, Egypt. Drs. Shahin, Niazy, and Attia as well as Ms. Teleb and Mr. Hamed are from the Department of Rheumatology and Rehabilitation. Drs. Yousef, Elmasry, Shalaby, Abdelkader, and Abdel-Halim as well as Ms. Elbendary and Mr. Mourad are from the Department of Dermatology. Dr. Hussein is from Al Kalaa Leprosarium Hospital, Cairo. Ms. Bahgat is from Al Omrania Leprosarium Hospital, Giza, Egypt.

The authors report no conflict of interest.

Correspondence: Mona R.E. Abdel-Halim, MD, Kasr Al Aini Hospital, Faculty of Medicine, Cairo University, Kasr Al Aini St, Cairo, Egypt 11562 (abdelhalimmona@gmail.com).

doi:10.12788/cutis.0417 
lymphohistiocytosis. The patient had no diabetes or hypertension and no history of deep vein thrombosis, stroke, or unintentional weight loss. No medications were taken prior to the onset of the skin lesions, and his family history was irrelevant.

General examination at the current presentation revealed a fever (temperature, $101.3{ }^{\circ} \mathrm{F}$ $\left[38.5^{\circ} \mathrm{C}\right]$ ), a normal heart rate $(90$ beats per minute), normal blood pressure $(120 / 80 \mathrm{mmHg})$, normal respiratory rate (14 breaths per minute), accentuated heart sounds, and normal vesicular breathing without adventitious sounds. He had saddle nose, loss of the outer third of the eyebrows, and marked reduction in the density of the eyelashes (madarosis). Bilateral pitting edema of the legs also was present. Neurologic examination revealed hypoesthesia in a glove-andstocking pattern, thickened peripheral nerves, and trophic changes over both hands; however, he had normal muscle power and deep reflexes. Joint examination revealed no abnormalities. Skin examination revealed widespread, reticulated, necrotizing, purpuric lesions on the arms, legs, abdomen, and ears, some associated with gangrenous ulcerations and hemorrhagic blisters. Scattered vasculitic ulcers and gangrenous patches were seen on the fingers. A gangrenous ulcer mimicking Fournier gangrene was seen involving the scrotal skin in addition to a gangrenous lesion on the glans penis (Figure 1-3). Unaffected skin appeared smooth, shiny, and edematous and showed no nodular lesions. Peripheral pulsations were intact.

Positive findings from a wide panel of laboratory investigations included an elevated erythrocyte sedimentation rate $(103 \mathrm{~mm}$ for the first hour [reference range, $0-22 \mathrm{~mm}])$, high $\mathrm{C}$-reactive protein $(50.7 \mathrm{mg} / \mathrm{L}$ [reference range, up to $6 \mathrm{mg} / \mathrm{L}$ ]), anemia (hemoglobin count, $7.3 \mathrm{~g} / \mathrm{dL}$ [reference range, 13.5-17.5 g/dL]), thrombocytopenia $\left(45 \times 10^{3} / \mathrm{mm}^{3}\right.$ [reference range, $\left.150 \times 10^{3} / \mathrm{mm}^{3}\right)$, low serum albumin $(2.3 \mathrm{~g} / \mathrm{dL}$ [reference range, 3.4-5.4 g/dL]), elevated IgG and IgM anticardiolipin antibodies (IgG, 21.4 IgG phospholipid [GPL] units [reference range, $<10$ IgG phospholipid (GPL) units]; IgM, 59.4 IgM phospholipid (MPL) units [reference range, $<7$ IgM phospholipid (MPL) units]), positive lupus anticoagulant panel test, elevated anti- $\beta 2$ glycoprotein antibodies (IgG, $17.5 \mu / \mathrm{mL}$ [reference range, $<8 \mu / \mathrm{mL}$ ]; IgM, $124.8 \mu / \mathrm{mL}$ [reference range, $<8 \mu / \mathrm{mL}$ ]), and low complement C3 (78 IU [reference range, 90-180 IU]). White blood cell count, liver and kidney functions, triglycerides, serum ferritin, and complement $\mathrm{C} 4$ all were normal. Hepatitis B surface antigen, hepatitis C viral antibody, HIV, antinuclear antibodies (ANA), antineutrophil cytoplasmic antibodies $\mathrm{C}$ and P (ANCA-C\&P), and venereal disease research laboratory tests all were negative.

Nerve conduction velocity showed axonal sensory polyneuropathy. Motor nerve conduction studies for median and ulnar nerves were within normal range. Lower-limb nerves assessment was limited by the

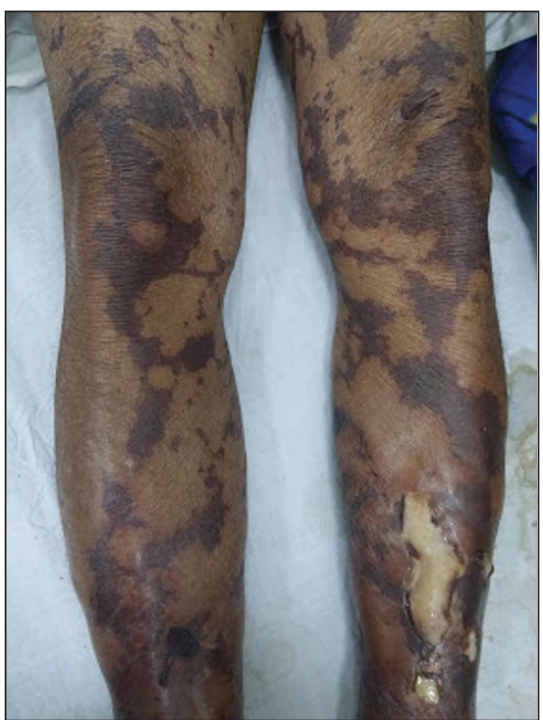

FIGURE 1. Necrotizing purpuric/gangrenous skin lesions involving the legs.

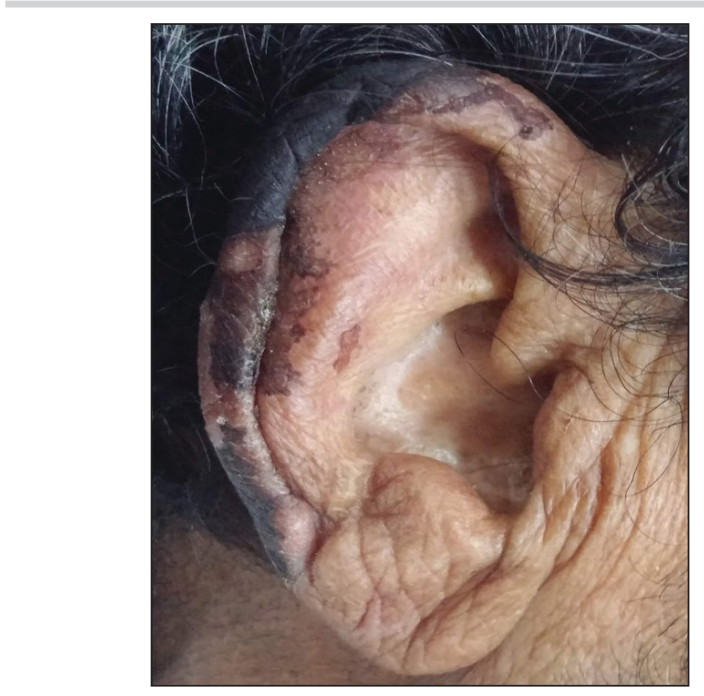

FIGURE 2. Necrotizing purpuric/gangrenous lesion involving the ear.

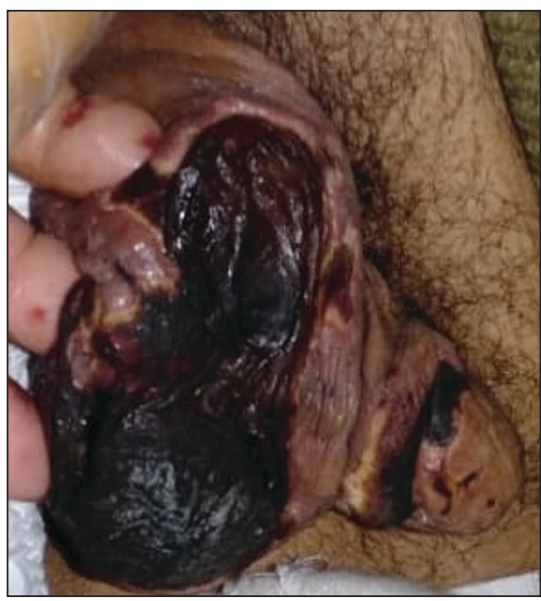

FIGURE 3. Founier gangrene-like scrotal lesion and penile gangrene. 
ulcerated areas and marked edema. Echocardiography was unremarkable. Arterial Doppler studies were only available for the upper limbs and were unremarkable.

A punch biopsy was taken from one of the necrotizing purpuric lesions on the legs, and histopathologic examination revealed foci of epidermal necrosis and subepidermal separation and superficial and deep perivascular and periadnexal infiltrates extending into the fat lobules. The infiltrates were mainly made up of foamy macrophages, and some contained globi (lepra cells), in addition to lymphocytes and many neutrophils with nuclear dust. Blood vessels in the superficial and deep dermis and in the subcutaneous fat showed fibrinoid necrosis in their walls with neutrophils infiltrating the walls and thrombi in the lumens (Figure 4). Modified Ziehl-Neelsen staining revealed clumps of acid-fast lepra bacilli inside vascular lumina and endothelial cell lining and within the foamy macrophages (Figure 5). Slit-skin smear examination was performed twice and yielded negative results. The slide and paraffin block of the already performed lymph node biopsy were retrieved. Examination revealed aggregates of foamy histiocytes surrounded by lymphocytes and plasma cells replacing normal lymphoid follicles. Modified Ziehl-Neelsen stain was performed, and clusters of acid-fast bacilli were detected within the foamy histiocytic infiltrate (Figure 6).

According to the results of the skin biopsy, the revised result of the lymph node biopsy, and the pattern of neurologic deficit together with clinical and laboratory correlation, the patient was diagnosed with diffuse nonnodular lepromatous leprosy presenting with Lucio phenomenon (Lucio leprosy) and associated with lepromatous lymphadenitis.

The patient received the following treatment: methylprednisolone $500 \mathrm{mg}$ (intravenous pulse therapy) followed by daily oral administration of prednisolone $10 \mathrm{mg}$, rifampicin $300 \mathrm{mg}$, dapsone $100 \mathrm{mg}$, clofazimine

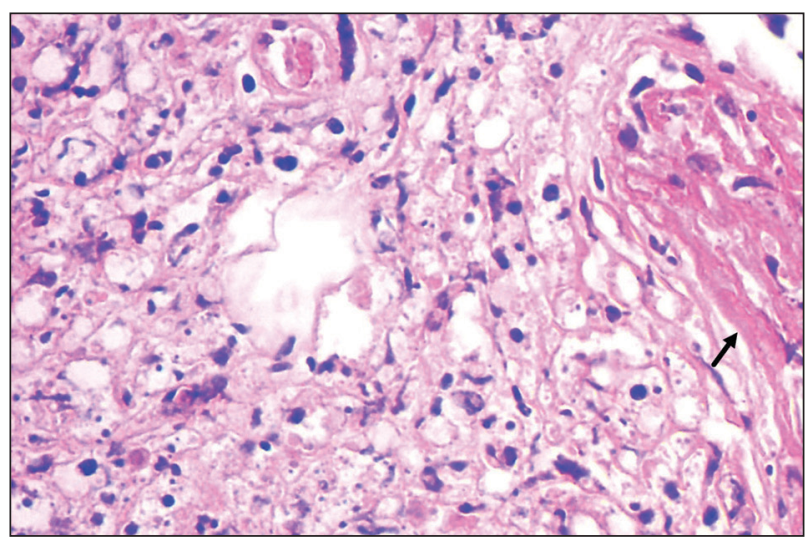

FIGURE 4. Photomicrograph depicting histopathologic changes of the skin biopsy taken from one of the necrotizing purpuric lesions on the legs in the form of dense collections of foamy histiocytes (lepra cells) in the subcutaneous fat with large vessel vasculitis and thrombosis (black arrow) $(\mathrm{H} \& \mathrm{E}$, original magnification $\times 100)$.
$100 \mathrm{mg}$, acetylsalicylic acid $150 \mathrm{mg}$, and enoxaparin sodium $80 \mathrm{mg}$. In addition, the scrotal Fournier gangrene-like lesion was treated by surgical debridement followed by vacuum therapy. By the second week after treatment, the gangrenous lesions of the fingers developed a line of demarcation, and the skin infarctions started to recede.

\section{Comment}

Despite a decrease in its prevalence through a World Health Organization (WHO)-empowered eradication program, leprosy still represents a health problem in endemic areas. ${ }^{1,2}$ It is characterized by a wide range of immune responses to Mycobacterium leprae, displaying a spectrum of clinical and histopathologic manifestations that vary from the tuberculoid or paucibacillary pole with a strong cell-mediated immune response and fewer organisms to the lepromatous or multibacillary pole with weaker cell-mediated immune response and higher loads of organisms. ${ }^{3}$ In addition to its well-known cutaneous and neurologic manifestations, leprosy can present with a variety of manifestations, including constitutional symptoms, musculoskeletal manifestations, and serologic abnormalities; thus, leprosy can mimic

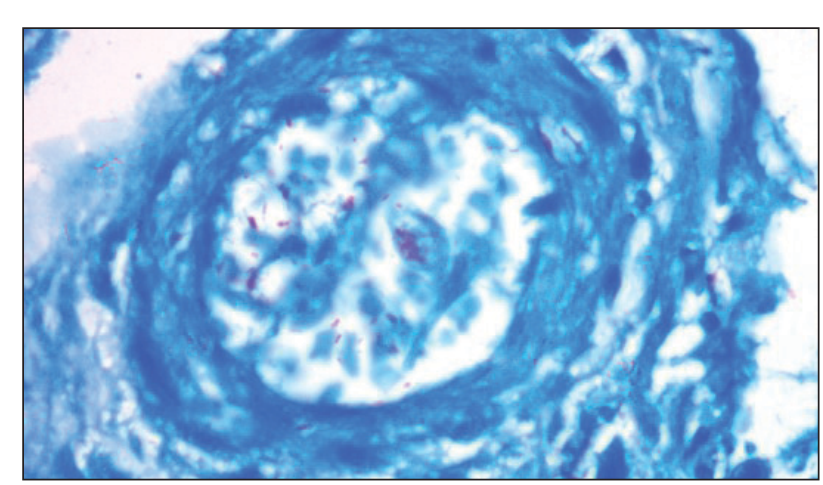

FIGURE 5. Photomicrograph depicting acid-fast bacilli within the lumen of a blood vessel and within endothelial cells in the skin biopsy taken from one of the necrotizing purpuric lesions in the legs (modified Ziehl-Neelsen, original magnification $\times 1000$, oil immersion).

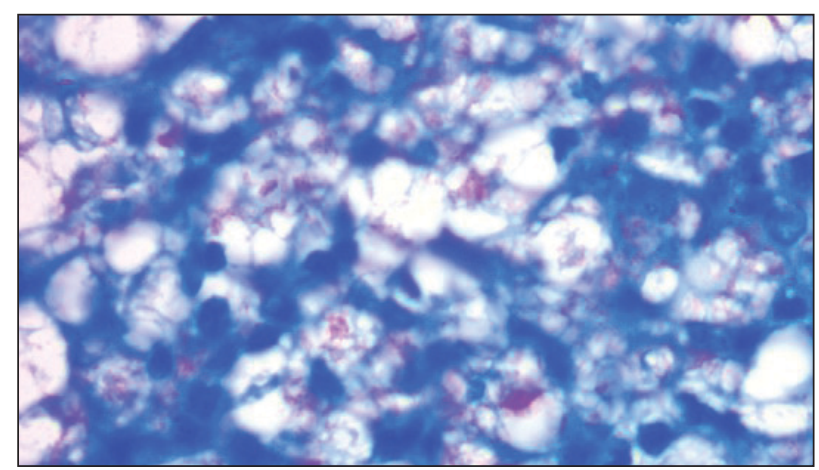

FIGURE 6. Photomicrograph depicting acid-fast bacilli in the lymph node biopsy (modified Ziehl-Neelsen, original magnification $\times 1000$, oil immersion). 
rheumatoid arthritis, spondyloarthritis, and vasculitisa pitfall that may result in misdiagnosis as a rheumatologic disorder..$^{3-7}$

The chronic course of leprosy can be disrupted by acute, immunologically mediated reactions known as lepra reactions, of which there are 3 types. ${ }^{8}$ Type I lepra reactions are cell mediated and occur mainly in patients with borderline disease, often representing an upgrade toward the tuberculoid pole; less often they represent a downgrade reaction. Nerves become painful and swollen with possible loss of function, and skin lesions become edematous and tender; sometimes arthritis develops. ${ }^{9}$ Type II lepra reactions, also known as erythema nodosum leprosum (ENL), occur in borderline lepromatous and lepromatous patients with a high bacillary load. They are characterized by fever, body aches, tender cutaneous/ subcutaneous nodules that may ulcerate, possible bullous lesions, painful nerve swellings, swollen joints, iritis, lymphadenitis, glomerulonephritis, epididymo-orchitis, and hepatic affection. Both immune-complex and delayed hypersensitivity reactions play a role in ENL. ${ }^{8,10}$ The third reaction is a rare aggressive type known as Lucio phenomenon or Lucio leprosy, which presents with irregular-shaped, angulated, or stellar necrotizing purpuric lesions (hemorrhagic infacrtions) developing mainly on the extremities. The lesions evolve into ulcers that heal with atrophic scarring., ${ }^{2,11}$ Lucio phenomenon develops as a result of thrombotic vascular occlusion secondary to massive invasion of vascular endothelial cells by lepra bacilli. ${ }^{2,11-14}$ Involvement of the scrotal skin, such as in our patient, is rare.

Lucio phenomenon mainly is seen in Mexico and Central America, and few cases have been documented in Cuba, South America, the United States, India, Polynesia, South Africa, and Southeast Asia. ${ }^{15-17}$ It specifically occurs in patients with untreated, diffuse, nonnodular lepromatous leprosy (pure and primitive diffuse lepromatous leprosy (DLL)/diffuse leprosy of Lucio and Latapí). This type of leprosy was first described by Lucio and Alvarado $^{18}$ in 1852 as a distinct form of lepromatous leprosy characterized by widespread and dense infiltration of the whole skin by lepra bacilli without the typical nodular lesions of leprosy, rendering its diagnosis challenging, especially in sporadic cases. Other manifestations of DLL include complete alopecia of the eyebrows and eyelashes, destructive rhinitis, and areas of anhidrosis and dyesthesia. ${ }^{2}$

Latapí and Chévez-Zomora ${ }^{19}$ defined Lucio phenomenon in 1948 as a form of histopathologic vasculitis restricted to patients with DLL. Histopathologically, in addition to the infiltration of the skin with acid-fast bacilli-laden foamy histiocytes, lesions of Lucio phenomenon show features of necrotizing (leukocytoclastic) vasculitis with fibrinoid necrosis ${ }^{20}$ or vascular thrombi with minimal perivascular lymphocytic infiltrate and no evidence of vasculitis. ${ }^{11}$ Medium to large vessels in the deep dermis and subcutaneous tissue show infiltration of their walls with a large number of macrophages laden with acid-fast bacilli. ${ }^{11}$ Cases with histopathologic features mimicking antiphospholipid syndrome with endothelial cell proliferation, thrombosis, and mild mononuclear cell infiltrate also may be seen. ${ }^{20}$ In all cases, ischemic epidermal necrosis is seen, as well as acid-fast bacilli, both singly and in clusters (globi) within endothelial cells and inside blood vessel lumina.

Although Lucio phenomenon initially was thought to be immune-complex mediated like ENL, it has been suggested that the main trigger is thrombotic vascular occlusion secondary to massive invasion of the vascular endothelial cells by the lepra bacilli, resulting in necrosis. ${ }^{14}$ Bacterial lipopolysaccharides promote the release of IL-1 and tumor necrosis factor $\alpha$, which in turn stimulate the production of prostaglandins, IL-6, and coagulation factor III, leading to vascular thrombosis and tissue necrosis. ${ }^{21,22}$ Moreover, antiphospholipid antibodies, which have been found to be induced in response to certain infectious agents in genetically predisposed individuals ${ }^{23}$ have been reported in patients with leprosy, mainly in association with lepromatous leprosy. The reported prevalence of anticardiolipin antibodies ranged from $37 \%$ to $98 \%$, whereas anti- $\beta 2$-glycoprotein I antibodies ranged from $3 \%$ to $19 \%$, and antiprothrombin antibodies ranged from $6 \%$ to $45 \% .{ }^{24,25}$ Antiphospholipid antibodies have been reported to play a role in the pathogenesis of Lucio phenomenon. ${ }^{11,13,15,26}$ Our case supports this hypothesis with positive anticardiolipin antibodies, anti- $\beta 2$ glycoprotein antibodies, and positive lupus anticoagulant.

In accordance with Curi et $\mathrm{al}^{2}{ }^{2}$ who reported 5 cases of DLL with Lucio phenomenon, our patient showed a similar presentation with positive inflammatory markers in association with a negative autoimmune profile (ANA, ANCA-C\&P) and negative venereal disease research laboratory test. It is important to mention that a positive autoimmune profile (ANA, ANCA-C\&P) can be present in leprotic patients, causing possible diagnostic confusion with collagen diseases. ${ }^{27,28}$

An interesting finding in our case was the negative slit-skin smear results. Although the specificity of slitskin smear is $100 \%$, as it directly demonstrates the presence of acid-fast bacilli, ${ }^{29}$ its sensitivity is low and varies from $10 \%$ to $50 \% .^{30}$ The detection of acid-fast bacilli in tissue sections is reported to be a better method for confirming the diagnosis of leprosy. ${ }^{31}$

The provisional impression of hemophagocytic lymphohistiocytosis in the lymph node biopsy in our patient was excluded upon detection of acid-fast bacilli in the foamy histiocytes infiltrating the lymph node; moreover, the normal serum lipids and serum ferritin argued against this diagnosis. ${ }^{32}$ Leprosy tends to involve the lymph nodes, particularly in borderline, borderline lepromatous, and lepromatous forms. ${ }^{33}$ The incidence of lymph node involvement accompanied by skin lesions with the presence of acid-fast bacilli in the lymph nodes is $92.2 \%{ }^{34}$ 
Our patient showed an excellent response to antileprotic treatment, which was administered according to the WHO multidrug therapy guidelines for multibacil-

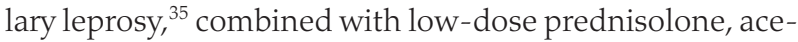
tylsalicylic acid, and anticoagulant treatment. Thalidomide and high-dose prednisolone $(60 \mathrm{mg} / \mathrm{d})$ combined with antileprotic treatment also have been reported to be successful in managing recurrent infarctions in leprosy. ${ }^{36}$ The Fournier-like gangrenous ulcer of the scrotum was managed by surgical debridement and vacuum therapy.

It is noteworthy that the WHO elimination goal for leprosy was to reduce the prevalence to less than 1 case per 10,000 population. Egypt is among the first countries in North Africa and the Middle East regions to achieve this target supervised by the National Leprosy Control Program as early as 1994; this was further reduced to 0.33 cases per 10,000 population in 2004, and reduced again in 2009; however, certain foci showed a prevalence rate more than the elimination target, particularly in the cities of Qena (1.12) and Sohag (2.47). ${ }^{37}$ Esna, where our patient is from, is an endemic area in Egypt. ${ }^{38}$

\section{Conclusion}

Leprosy is a great mimicker of many connective tissue diseases, including vasculitis. Antiphospholipid antibodies are involved in Lucio phenomenon. Recognition of Lucio phenomenon is important to initiate prompt treatment and avoid morbidity and mortality. We report a rare case of diffuse nonnodular lepromatous leprosy in Egypt in which Lucio phenomenon was the first diagnostic presentation. Scrotal involvement with Lucio phenomenon was not previously reported in any case of Lucio leprosy.

\section{REFERENCES}

1. World Health Organization. World Health Statistics: 2011. World Health Organization; 2011. https://www.who.int/gho/publications /world_health_statistics/EN_WHS2011_Full.pdf

2. Curi PF, Villaroel JS, Migliore N, et al. Lucio's phenomenon: report of five cases. Clin Rheumatol. 2016;35:1397-1401.

3. Shrestha B, LiYQ, Fu P. Leprosy mimics adult onset Still's disease in a Chinese patient. Egypt Rheumatol. 2018;40:217-220.

4. Prasad S, Misra R, Aggarwal A, et al. Leprosy revealed in a rheumatology clinic: a case series. Int J Rheum Dis. 2013;16:129-133.

5. Chao G, Fang L, Lu C. Leprosy with ANA positive mistaken for connective tissue disease. Clin Rheumatol. 2013;32:645-648.

6. Chauhan S, Wakhlu A, Agarwal V. Arthritis in leprosy. Rheumatology. 2010;49:2237-2242

7. Rath D, Bhargava S, Kundu BK. Leprosy mimicking common rheumatologic entities: a trial for the clinician in the era of biologics. Case Rep Rheumatol. 2014;2014:429698.

8. Cuevas J, Rodríguez-Peralto JL, Carrillo R, et al. Erythema nodosum leprosum: reactional leprosy. Semin Cutan Med Surg. 2007;26:126-130

9. Henriques CC, Lopéz B, Mestre T, et al. Leprosy and rheumatoid arthritis: consequence or association? BMJ Case Rep. 2012;13:1-4.

10. Vázquez-Botet M, Sánchez JL. Erythema nodosum leprosum. Int J Dermatol. 1987;26:436-437.

11. Nunzie E, Ortega Cabrera LV, Macanchi Moncayo FM, et al. Lucio leprosy with Lucio's phenomenon, digital gangrene and anticardiolipin antibodies. Lepr Rev. 2014;85:194-200.
12. Salvi S, Chopra A. Leprosy in a rheumatology setting: a challenging mimic to expose. Clin Rheumatol. 2013;32:1557-1563.

13. Azulay-Abulafia L, Pereira SL, Hardmann D, et al. Lucio phenomenon. vasculitis or occlusive vasculopathy? Hautarzt. 2006;57:1101-1105.

14. Benard G, Sakai-Valente NY, Bianconcini Trindade MA. Concomittant Lucio phenomenon and erythema nodosum in a leprosy patient: clues for their distinct pathogenesis. Am J Dermatopathol. 2009;31:288-292.

15. Rocha RH, Emerich PS, Diniz LM, et al. Lucio's phenomenon: exuberant case report and review of Brazilian cases. An Bras Dermatol. 2016;91(suppl 5):S60-S63.

16. Costa IM, Kawano LB, Pereira CP, et al. Lucio's phenomenon: a case report and review of the literature. Int J Dermatol. 2005;44:566-571.

17. Kumari R, Thappa DM, Basu D. A fatal case of Lucio phenomenon from India. Dermatol Online J. 2008;14:10.

18. Lucio R, Alvarado I. Opúsculo Sobre el Mal de San Lázaro o Elefantiasis de los Griegos. M. Murguía; 1852

19. Latapí F, Chévez-Zamora A. The "spotted" leprosy of Lucio: an introduction to its clinical and histological study. Int J Lepr. 1948;16:421-437.

20. Vargas OF. Diffuse leprosy of Lucio and Latapí: a histologic study. Lepr Rev. 2007;78:248-260.

21. Latapí FR, Chevez-Zamora A. La lepra manchada de Lucio. Rev Dermatol Mex. 1978;22:102-107

22. Monteiro R, Abreu MA, Tiezzi MG, et al. Fenômeno de Lúcio: mais um caso relatado no Brasil. An Bras Dermatol. 2012;87:296-300.

23. Gharavi EE, Chaimovich H, Cucucrull E, et al. Induction of antiphospholipid antibodies by immunization with synthetic bacterial \& viral peptides. Lupus. 1999;8:449-455.

24. de Larrañaga GF, Forastiero RR, Martinuzzo ME, et al. High prevalence of antiphospholipid antibodies in leprosy: evaluation of antigen reactivity. Lupus. 2000;9:594-600.

25. Loizou S, Singh S, Wypkema E, et al. Anticardiolipin, antibeta(2)-glycoprotein I and antiprothrombin antibodies in black South African patients with infectious disease. Ann Rheum Dis. 2003;62:1106-1111.

26. Akerkar SM, Bichile LS. Leprosy \& gangrene: a rare association; role of antiphospholipid antibodies. BMC Infect Dis. 2005,5:74.

27. Horta-Baas G, Hernández-Cabrera MF, Barile-Fabris LA, et al. Multibacillary leprosy mimicking systemic lupus erythematosus: case report and literature review. Lupus. 2015;24:1095-1102.

28. Pradhan V, Badakere SS, Shankar KU. Increased incidence of cytoplasmic ANCA (cANCA) and other auto antibodies in leprosy patients from western India. Lepr Rev. 2004;75:50-56.

29. Oskam L. Diagnosis and classification of leprosy. Lepr Rev. 2002;73:17-26.

30. Rao PN. Recent advances in the control programs and therapy of leprosy. Indian J Dermatol Venereol Leprol. 2004;70:269-276.

31. Rao PN, Pratap D, Ramana Reddy AV, et al. Evaluation of leprosy patients with 1 to 5 skin lesions with relevance to their grouping into paucibacillary or multibacillary disease. Indian J Dermatol Venereol Leprol. 2006;72:207-210.

32. Rosado FGN, Kim AS. Hemophagocytic lymphohistiocytosis. an update on diagnosis and pathogenesis. Am I Clin Pathol. 2013;139:713-727.

33. Kar HK, Mohanty HC, Mohanty GN, et al. Clinicopathological study of lymph node involvement in leprosy. Lepr India. 1983;55:725-738.

34. Gupta JC, Panda PK, Shrivastava KK, et al. A histopathologic study of lymph nodes in 43 cases of leprosy. Lepr India. 1978;50:196-203.

35. WHO Expert Committee on Leprosy. Seventh Report. World Health Organization; 1998. https://apps.who.int/iris/bitstream /handle/10665/42060/WHO_TRS_874.pdf? sequence $=1$ \&isAllowed=y

36. Misra DP, Parida JR, Chowdhury AC, et al. Lepra reaction with Lucio phenomenon mimicking cutaneous vasculitis. Case Rep Immunol. 2014;2014:641989.

37. Amer A, Mansour A. Epidemiological study of leprosy in Egypt: 2005-2009. Egypt J Dermatol Venereol. 2014;34:70-73.

38. World Health Organization. Screening campaign aims to eliminate leprosy in Egypt. Published May 9, 2018. Accessed September 8, 2021. http://www.emro.who.int/egy/egypt-events/last-miless-activities -on-eliminating-leprosy-from-egypt.html 